\title{
Analysis of Electromotive Force Characteristics for Electromagnetic Energy Harvester using Ferrofluid
}

\author{
Young Sun Kim* \\ Department of Electrical and Electronic Engineering, Joongbu University, Goyang 412-480, Korea
}

(Received 6 April 2015, Received in final form 12 June 2015, Accepted 16 July 2015)

\begin{abstract}
This paper investigates the concept and implementation of an energy harvesting device using a ferrofluid sloshing movement to generate an electromotive force (EMF). Ferrofluids are often applied to energy harvesting devices because they have both magnetic properties and fluidity, and they behave similarly to a soft ferromagnetic substance. In addition, a ferrofluid can change its shape freely and generate an EMF from small vibrations. The existing energy harvesting techniques, for example those using piezoelectric and thermoelectric devices, generate minimal electric power, as low as a few micro-watts. Through flow analysis of ferrofluids and examination of the magnetic circuit characteristics of the resultant electromagnetic system, an energy harvester model based on an electromagnetic field generated by a ferrofluid is developed and proposed. The feasibility of the proposed scheme is demonstrated and its EMF characteristics are discussed based on experimental data.
\end{abstract}

Keywords : electromotive force, energy harvester, ferrofluid, reluctance, sloshing, vibration

\section{Introduction}

Energy harvesting is the process by which energy is derived from external sources and stored in small devices used in wearable electronics. The energy source for energy harvesters is present as ambient background and is therefore freely available. Examples of sources include vibration, wind power, wave power, and thermal temperature gradients. Here, we focus on vibration as a representative source which can be converted into electric power in the environment.

Mechanical vibration is a very attractive energy source due to its abundance [1-4]. Some vibration sources in the environment include electric motor rotation, wind power, wave power, vehicle motion, human movement, and seismic vibrations all of which vary widely in both frequency and amplitude. Vibration energy can be harvested using several methods, such as piezoelectric, electrostatic, and electromagnetic transduction. Piezoelectric energy harvesters provide higher output voltages and are more efficient for relatively high vibration frequencies. Electromagnetic energy harvesters are better candidates for low frequency vibrations [5], however, their generated peak

CThe Korean Magnetics Society. All rights reserved.

*Corresponding author: Tel: +82-31-8075-1631

Fax: +82-31-8075-1530, e-mail: yskim@joongbu.ac.kr output voltage is relatively low. In addition, the design of the applied power electronics and electromagnetic systems in the device are crucial in delivering the harvested power to the load efficiently. Various harvester configurations have been studied, with the basic concept remaining the same. The core principle is that external vibrations are used to set a permanent magnet in motion relative to a stationary induced voltage coil. As per Faraday's law, the change of magnetic flux produces a current in the induced voltage coil which can then drive an electrical device.

This paper investigates a ferrofluid (magnetic fluid) based electromagnetic energy harvester which has ferromagnetic back yoke. In the past, studies by Bibo et al. are including an induced voltage according to magnetic field intensity and frequencies of external vibration. However, research of this kind is not considered a back yoke which makes closed magnetic flux path [6]. Configuration of closed magnetic circuit increases the amount of magnetic flux linkage passing ferrofluid by changing the operating point of the permanent magnet. This method is frequently used to increase the magnetic flux for magnetic sensor system [7, 8]. Use of back yoke is necessary because the amount of magnetic flux generated by energy harvester is small. It is the cause of the increasing sensitivity of flux variation.

In this paper, the ferromagnetic core is used as a back yoke for an electromagnetic energy harvester and ferrofluid 
is adopt as a moving magnetic material. Ferrofluids are colloidal liquids made of nanoscale permanent magnetic dipoles and a ferrofluid can change shape freely and generates an electromotive force (EMF) from small vibrations. Ferrofluids are often applied to energy harvesting devices, because they have fluidity and magnetic properties and behave like soft ferromagnetic substance [911]. The existing energy harvesting techniques mentioned previously, along with thermoelectric devices, generate minimal electric power only, as little as a few micro-watts. Here, through studies on the flow analysis of ferrofluids and the magnetic circuit characteristics of the resultant electromagnetic system, an energy harvester model based on an electromagnetic field generated by a ferrofluid is developed and proposed. Then, the feasibility of the proposed method is shown and its EMF characteristics are discussed through the use of experimental data.

\section{Harvester Design with Experimental Setup}

Fig. 1 is a diagram of the proposed energy harvester using a ferrofluid with an induced EMF coil in its core. The motion of the sloshing ferrofluid in the container changes the magnetic flux generated by the permanent magnet and creates a time-varying magnetic flux. This flux induces an EMF in a coil wound around the ferromagnetic core, thus generating an electric current.

To investigate the feasibility of the proposed concept, the experimental setup shown in Fig. 2 is constructed. A rectangular container with an area of $100 \times 60 \mathrm{~mm}$ and a

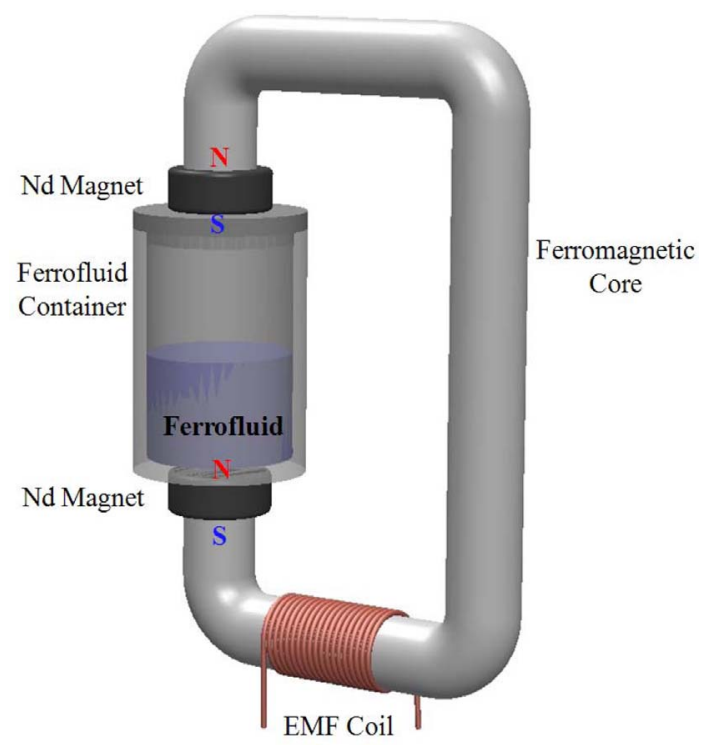

Fig. 1. (Color online) Design concept of electromagnetic energy harvester using ferrofluid sloshing motion.

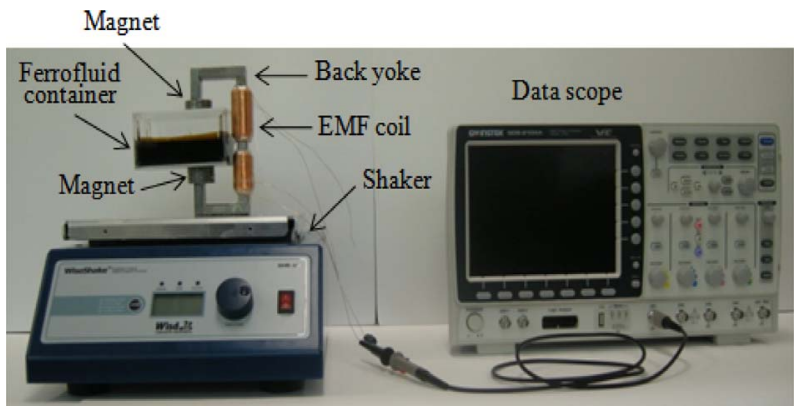

Fig. 2. (Color online) Full experimental setup for inducing EMF in electromagnetic energy harvester using ferrofluid.

height of $50 \mathrm{~mm}$ is mounted on a mechanical shaker, which supplies vibrations of different speeds, ranging from 0-300 rpm. This vibration causes a sloshing motion of the ferrofluid with a frequency range of $0-5 \mathrm{~Hz}$. Two groups of permanent magnets generate a magnetic flux at the top and bottom of the ferrofluid container. This flux travels through the ferromagnetic core and the ferrofluid region. Ferrofluids are stable colloidal suspensions of single-domain magnetic nanoparticles in a carrier fluid such as oil or water. The nanoparticles are usually ferroor ferrimagnetic particles with typical diameters of order $10 \mathrm{~nm}$ coated with a surfactant layer of 1 to $2 \mathrm{~nm}$. There are two kinds of ferrofluid that are widely known and the material properties of these ferrofluids are given in Table 1. Most of these values have been taken from measurements by Elborai and $\mathrm{He}$, as documented in their respective theses $[10,11]$. The first ferrofluid, MSGW11, is water-based, while the second, EFH1, is hydrocarbon-oilbased ferrofluid. The latter is used in this experiment because of relatively large magnetic permeability.

To construct a magnetic circuit, a closed magnetic flux circuit is constructed using a back iron yoke of ferromagnetic material known as 'Steel $45 \mathrm{C}$ ', consisting of two winding groups of 600 turns around the iron core. It is possible to reduce the leakage flux using a back iron yoke around both sides of the magnet and the EMF coil.

Table 1. Values of Physical, Mechanical, and Magnetic Properties for the Two Ferrofluids Used in the Electromagnetic Harvester.

\begin{tabular}{lcc}
\hline \hline \multicolumn{1}{c}{ Ferrofluid } & MSGW11 & EFH1 \\
\hline Mass density $\left[\mathrm{kg} / \mathrm{m}^{3}\right]$ & 1,200 & 1,221 \\
Viscosity [cP] & 2.02 & 7.27 \\
Saturation magnetization [G] & 153.9 & 421.2 \\
Relative permeability & 1.56 & 2.59 \\
Magnetic susceptibility & 0.56 & 1.59 \\
Volume fraction \% & 2.75 & 7.52 \\
Avg. particle diameter [nm] & 7.9 & 10.6 \\
\hline
\end{tabular}




\section{Induced Electromotive Force}

The magnetic flux is altered by changing the magnitude of the magnetic induction generated by the sloshing motion of the ferrofluid, which is located between the lower and upper magnet surfaces of the container, as shown in Fig. 2. Thus, the derivative of the linkage flux to the EMF coil generates an electromotive force.

The experimental conditions for a given volume of ferrofluid and a specific magnetic induction generated by the permanent magnet are shown in Table 2. The resultant EMF by the external vibrations (ranging $0-5 \mathrm{~Hz}$ ) are measured using an oscilloscope for each conditions. Fig. 3 gives the profile of the electromotive force of the EMF coil with respect to the vibration speed, in response to various frequencies. The generated EMF waveforms have a unique frequency and small amplitude. The frequencies by trend line of the induced EMF are 3, 4, and $5 \mathrm{~Hz}$ and

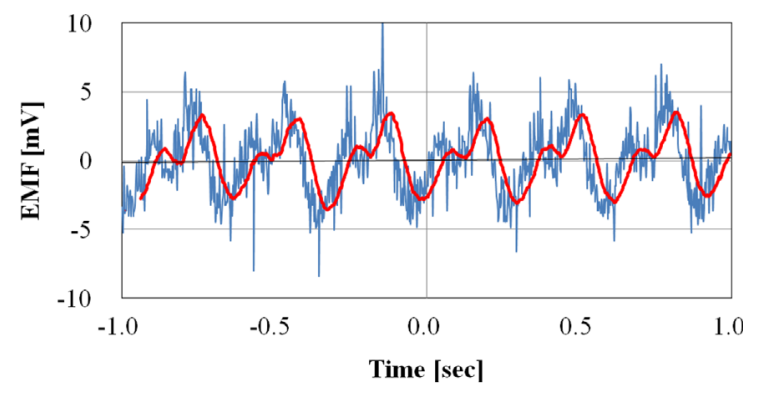

(a) Vibration frequency : $3 \mathrm{~Hz}$

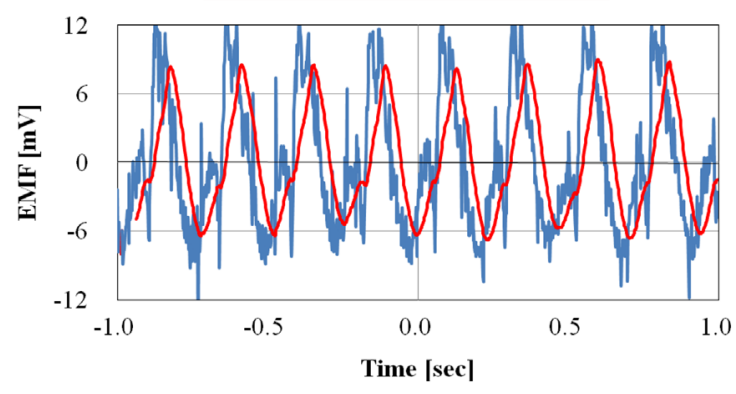

(b) Vibration frequency : $4 \mathrm{~Hz}$

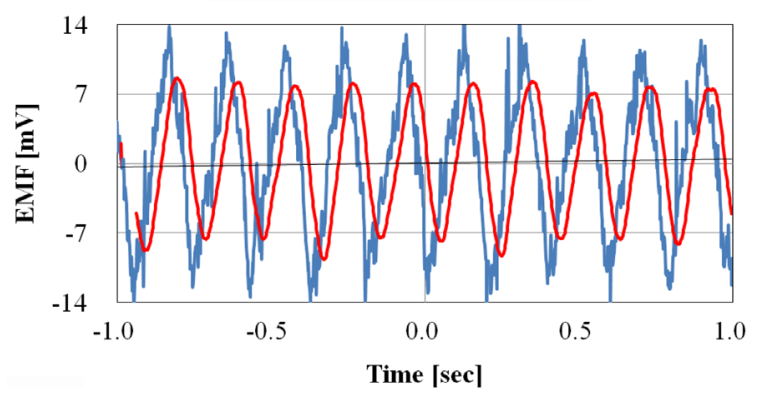

(c) Vibration frequency : $5 \mathrm{~Hz}$

Fig. 3. (Color online) Instantaneous values of EMF generated by sloshing motion of ferrofluid for several external vibration frequencies.
Table 2. Experimental Conditions for Energy Harvester

\begin{tabular}{cccc}
\hline \hline Factor & Model & Quan. \& mag. & Remark \\
\hline \multirow{2}{*}{ Ferrofluid } & F1 & $100 \mathrm{ml}$ & Container volume : \\
volume & F2 & $150 \mathrm{ml}$ & $300 \mathrm{ml}$ \\
& F3 & $200 \mathrm{ml}$ & \\
\hline \multirow{2}{*}{ Magnetic flux } & M1 & $220 \mathrm{mT}$ & at the upper magnet \\
density & M2 & $310 \mathrm{mT}$ & surface \\
& M3 & $400 \mathrm{mT}$ & \\
\hline
\end{tabular}

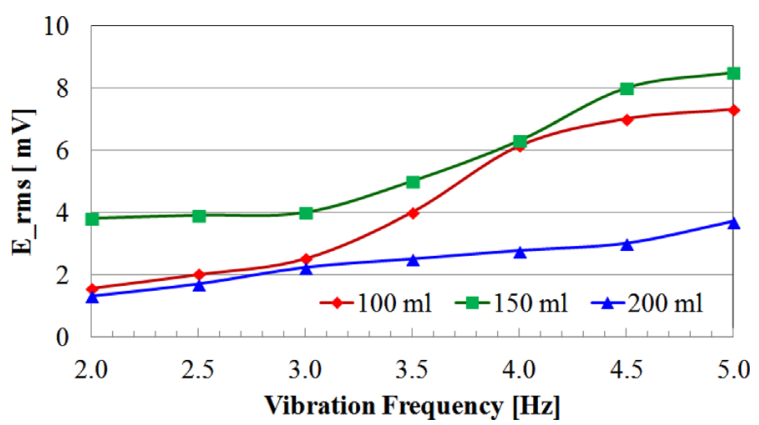

Fig. 4. (Color online) RMS values of the EMF against vibration frequency for a varying ferrofluid volume. The ferrofluid volumes are $1 / 3,1 / 2$, and $2 / 3$ of the overall container volume.

the magnitude of the induced voltages increase in proportion to the increased vibration speed.

The root-mean-square values of the induced electromotive force are shown in Fig. 4. The results demonstrate that variations in the ferrofluid volume influence the induced voltage of the harvester. The EMF value in the $100 \mathrm{ml}$ ferrofluid case is larger than that of $200 \mathrm{ml}$ case, as a result of the reduced shaking of the ferrofluid. In the $100 \mathrm{ml}$ case, the EMF value is increased rapidly about 7 $\mathrm{mV}$ near the $4 \mathrm{~Hz}$. Thus, it can be concluded that the best ferrofluid volume for this harvester design is at approximately one third of the overall container volume. Of course, this is limited in the case of the proposed harvester dimension.

Fig. 5 shows alternative arrangements of the permanent magnets so that the ferrofluid in the container experiences a variety of magnetic fluxes. The $\boldsymbol{B}_{z}$ profiles in each figure represent the magnetic flux density along the normal center line due to the effects of the lower and upper permanent magnets. Because the ferrofluid adheres to the lower magnet (as a result of the gravitational force), variation in the magnetic induction is achieved simply by altering the number of upper magnets. The magnitudes of the magnetic flux densities at the magnet surfaces in each of the arrangements are shown in Fig. 5 are 57, 82, and $94 \mathrm{mT}$, respectively, when the magnets are docked in the energy harvester. 


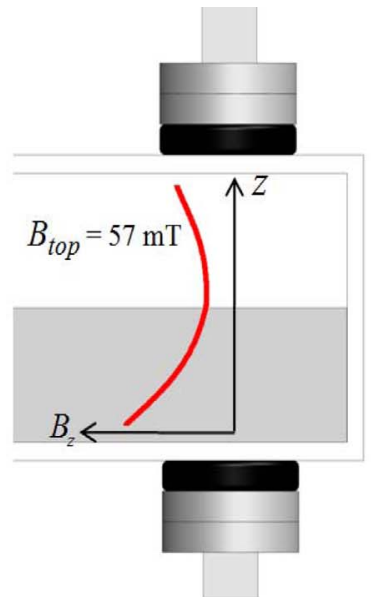

(a)

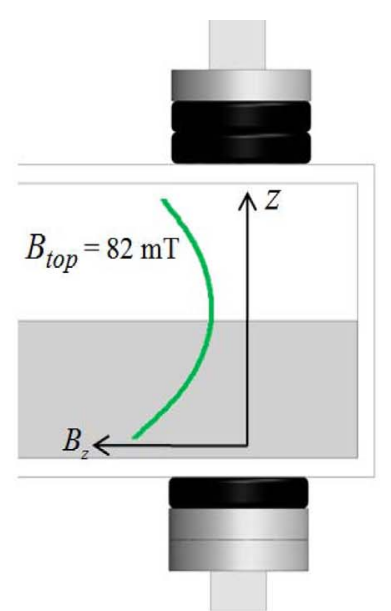

(b)

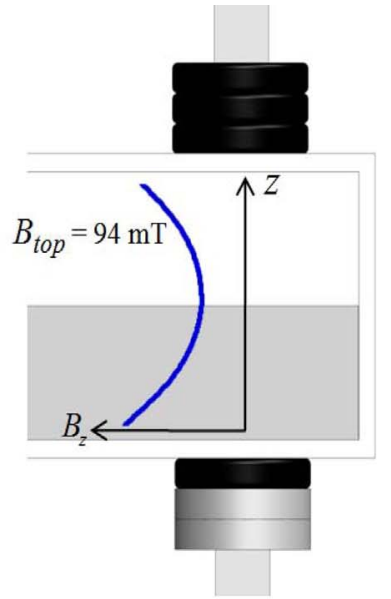

(c)

Fig. 5. (Color online) Three kinds of magnet arrangement and respective magnetic flux density profiles along the normal center of the lower magnet to upper magnet. Model names are (a) M1, (b) M2, and M3.

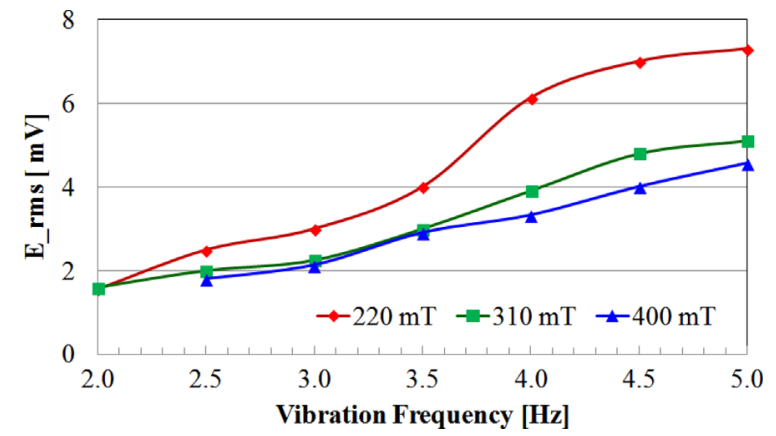

Fig. 6. (Color online) RMS values of the EMF according to the vibration frequency for the varying magnetic induction of upper magnet surface.

Fig. 6 illustrates the EMF profiles of the energy harvester with respect to vibration speed. The bigger the magnetic induction, the smaller the EMF values, because a large amount of ferrofluid sticks to the end of the magnet pole in the case of the three-piece magnet. Because the ferrofluid clings to the magnet pole, the sloshing motion is reduced, which in turn causes a decrease in the reluctance variation of the ferrofluid region.

\section{Numerical Analysis}

To determine the EMF characteristics of the proposed energy harvester, 2-D finite element analysis on the magnetostatic field is performed using a specially selfdeveloped program. Fig. 7 is a schematic diagram illustrating the magnetic flux linkage to the EMF coil. The magnetic flux, $\phi$, generated by the permanent magnet is also expressed by the magnetic vector potential as

$$
\phi= \pm N \int_{\text {surface }} \mathbf{B}(x, y) \cdot \mathbf{d s}= \pm N \int_{\text {coil }} \mathbf{A}(x, y) \cdot \mathbf{d} \mathbf{l}
$$

where, $\mathbf{B}$ is the magnetic flux density passing through the closed loop, $\mathbf{A}$ is the magnetic vector potential of the EMF coil region, and the ' \pm ' indicates the direction of the induced EMF.

The EMF is calculated by differentiating the magnetic flux linkage with respect to time. The induced voltage of the EMF coil, $E_{a}$, is expressed as a differential equation of the flux linkage for a closed loop as

$$
E_{a}=N \frac{d \phi}{d t}=N \frac{d}{d t} \int_{s} \mathbf{B} \cdot \mathbf{d} \mathbf{s}=N \frac{d}{d t} \oint_{c} \mathbf{A} \cdot \mathbf{d} \mathbf{l}
$$

where, $N$ is the number of turns, $\mathbf{B}$ is the linkage flux density, and $s$ is the winding area. Using Stokes' theorem, the EMF formula is then rewritten as

$$
E_{a}=l \frac{N}{S_{a}} \frac{d}{d t} \int_{S_{a^{+}}} A_{z} d s-\int_{S_{a^{-}}} A_{z} d s
$$

where, $l$ is the axis length of the coil, $S_{a}^{+}$is the positive winding area, and $S_{a}^{-}$is the negative winding area. Finally, Eq. (3) is modified to become

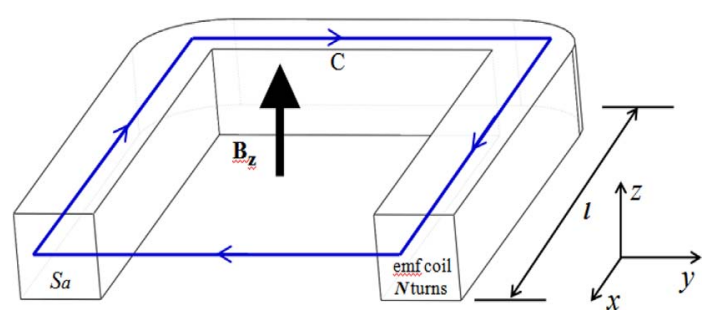

Fig. 7. (Color online) Schematic diagram of magnetic flux linkage to EMF coil. 
Table 3. Specifications for Electromagnetic Field Analysis of Harvester

\begin{tabular}{ccc}
\hline \hline Part & Property & Value \\
\hline \multirow{2}{*}{ Permanent Magnet } & Diameter \& thickness & $25 \mathrm{~mm}, 5 \mathrm{~mm}$ \\
& Residual induction & $220 \mathrm{mT}$ \\
\hline \multirow{2}{*}{ EFH1 by Ferrotec ${ }^{\circledR}$} & Relative Permeability & 1.56 \\
& Amount & $150 \mathrm{ml}$ \\
\hline \multirow{2}{*}{ on core } & Type & Steel $45 \mathrm{C}$ \\
& Relative Permeability & 2,000 \\
\hline \multirow{2}{*}{ EMF Coil } & Resistance 1 & $61.3 \Omega$ \\
& Inductance & $74.3 \mathrm{mH}$ \\
\hline
\end{tabular}

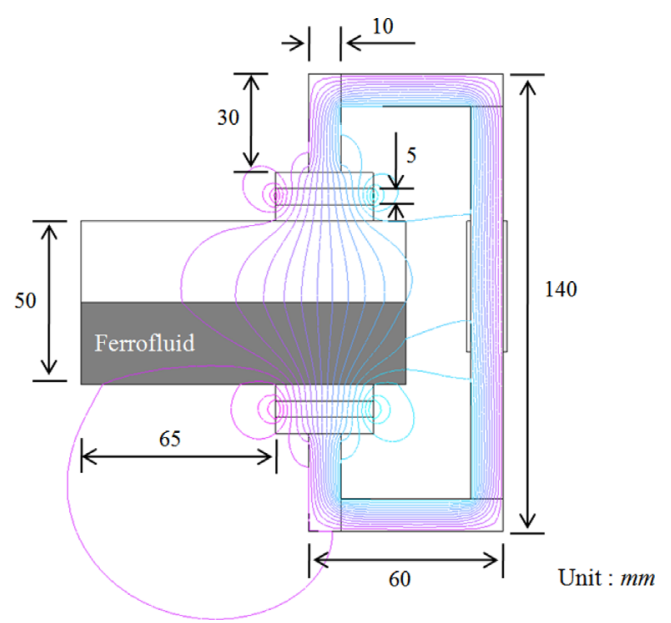

Fig. 8. (Color online) Schematic diagram of magnetic flux linkage to EMF coil with $\mathrm{N}$ turns at the energy harvester core.

$$
E_{a}=l \frac{N}{S_{a}} \frac{d}{d t}\left(A_{a+}{ }^{t}-A_{a^{-}}{ }^{t}\right)
$$

where, $A_{a^{+}}{ }^{t}$ and $A_{a-}{ }^{t}$ denote the mean values of the magnetic vector potentials at the positive and negative windings, respectively.

Through the finite element analysis of the electromag-

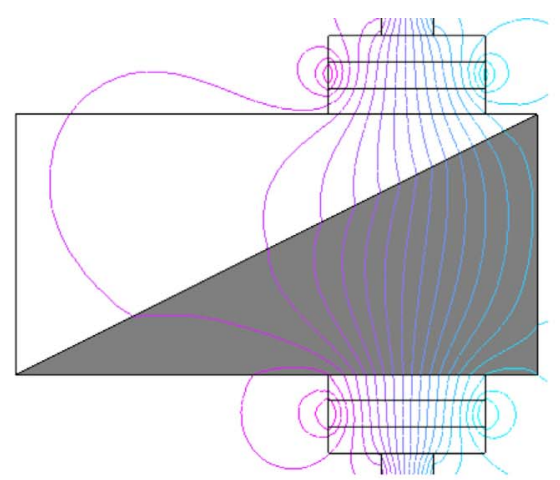

(a) netic field of the energy harvester with varying parameters (such as dimensions and material properties), the optimal shape of the back iron yoke is obtained. Fig. 8 illustrates the dimensions and equi-potential line to understand the magnetic field distribution using the information from Table 3. It can be seen that the magnetic flux generated in the lower magnet travels to the upper magnet through the ferrofluid in the container with minimal leakage flux.

The external vibrations used in the experiment are artificially applied to the energy harvester by the mechanical shaker. This has a reciprocating motion and the vibration speeds are displayed in units of $\mathrm{Hz}$. The vibration half-period used in the numerical simulation is calculated using Eq. (5) below, and Fig. 9 shows the sloshing phenomena modeling scheme for the ferrofluid in the container. This simulation is used to determine the parameters that generate the maximum EMF. In this setup, the reluctance difference in the magnetic circuit between two states is maximized. It is found that the calculated values of the EMF analysis are quite different to the experimental results, with approximate errors over tens of $\mathrm{mV}$ in the overall speed range. This is caused by the sticking phenomena, as the ferrofluid adhesion to the magnet pole cannot be taken into account in the numerical simulation.

$$
T_{\text {half }}=\frac{1}{r p m / 60} \times \frac{1}{2}
$$

\section{Conclusion}

This work demonstrates the feasibility of the proposed electromagnetic energy harvester using a ferrofluid as the dynamic component. The characteristics of the EMF in the induced coil around the yoke in response to changes in the ferrofluid volume and the permanent magnet strength are investigated. We then construct an initial prototype of

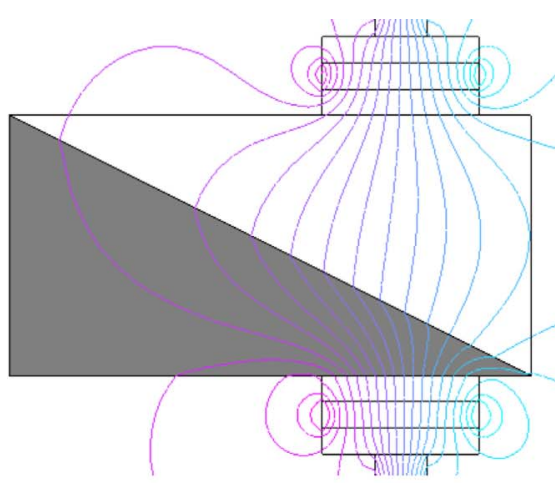

(b)

Fig. 9. (Color online) Modeling of sloshing phenomena for the ferrofluid in the container to generate maximum EMF. 
the appropriate dimensions and material properties of the proposed electromagnetic energy harvester and conduct a numerical analysis of the electromagnetic field. Although the resultant power levels are small compared with those of the traditional energy harvester using magnet vibration, the proposed energy harvester has the following unique advantages: 1) Applicability to low frequency and small external vibration amplitude scenarios, and 2) increased EMF efficiency due to the use of a back iron yoke. Future avenues of research include determining the optimal parameters to further improve the harvester's power generation performance.

\section{Acknowledgment}

This research was supported by Basic Science Research Program through the National Research Foundation of Korea (NRF) funded by the Ministry of Education, Science and Technology (2012R1A1A1040410).

\section{References}

[1] S. Roundy, P. K. Wright, and J. Rabaey, Computer Commun. 26, 1131 (2003).
[2] S. Priya, Appl. Phys. Lett. 87, 184101 (2005).

[3] D. Jia, J. Liu, and Y. Zhou, Phys. Lett. A 373, 1305 (2009).

[4] A. Munaz, B. Lee, and G. Chung, Sensor and Actuators A 201, 134 (2013).

[5] A. Rahimi, Ö. Zorlu, A. Muhtaroglu, and H. Külah, Procedia Engineering 25, 215 (2011).

[6] A. Bibo, R. Masana, A. King, G. Li, and M. F. Daqaq, Phys. Lett. A 376, 2163 (2012).

[7] Rajesh Keshwani and Shiben Bhattacharya, Sensor Rev. 29, 355 (2009).

[8] M. Takagishi, K. Komaki, and H. Fuminori, IEEE Trans. Magn. 33, 2821 (1997).

[9] R. E. Rosensweig, Ferrohydrodunamics, Dover ed., New York: Cambridge University Press (1985).

[10] S. Elborai, Ferrofluid surface and volume flows in uniform rotating magnetic fields, Ph.D thesis, Massachusetts Institute of Technology, Cambridge, MA (2006).

[11] X. He, Ferrohydrodynamic flows in uniform and nonuniform rotating magnetic fields, Ph.D thesis, Dept. of Electrical Engineering and Computer Science, Massachusetts Institute of Technology, Cambridge, MA (2006). 